\title{
Space Suit Joint Torque Measurement Method Validation
}

\author{
Dana Valish ${ }^{1}$ and Karina Eversley ${ }^{2}$ \\ NASA Johnson Space Center, Houston, TX, 77058
}

\begin{abstract}
In 2009 and early 2010, a test method was developed and performed to quantify the torque required to manipulate joints in several existing operational and prototype space suits. This was done in an effort to develop joint torque requirements appropriate for a new Constellation Program space suit system. The same test method was levied on the Constellation space suit contractors to verify that their suit design met the requirements. However, because the original test was set up and conducted by a single test operator, there was some question as to whether this method was repeatable enough to be considered a standard verification method for Constellation or other future development programs. To validate the method itself, a representative subset of the previous test was repeated using the same information that would be available to space suit contractors, but set up and conducted by someone not familiar with the previous test. The resultant data were compared using graphical and statistical analysis; the results indicated a significant variance in values reported for a subset of the re-tested joints. Potential variables that could have affected the data were identified and a third round of testing was conducted in an attempt to eliminate and/or quantify the effects of these variables. The results of the third test effort was used to determine that the proposed joint torque methodology can be applied to future space suit development contracts.
\end{abstract}

$\begin{array}{ll} & \\ \text { ERD } & =\text { Element Requirements Document } \\ \text { ESPO } & =\text { Extravehicular Activity Systems Project Office } \\ \text { EVA } & =\text { Extravehicular Activity } \\ \text { psid } & =\text { pounds per square inch } \\ \text { ROM } & =\text { range of motion } \\ \text { TC1 } & =\text { test conductor } 1 \\ \text { TC2 } & =\text { test conductor } 2 \\ \text { TMG } & =\text { Thermal Micrometeoroid Garment }\end{array}$

\section{Introduction}

TN 2009 and early 2010, a test method was developed and 1 performed to quantify the torque required to manipulate joints in several existing operational and prototype space suits. This was done in an effort to develop joint torque requirements appropriate for a new Constellation Program space suit system. The method, called the "modified fish-scale method" ${ }^{1}$, consisted of applying force externally to pressurized, unmanned suit joints as they were manipulated through their full range of motion (ROM). This force was measured using a load cell and converted to torque based on a distance measurement from the location of the applied force to the axis of joint rotation (estimated location of human joint axis). The angle of the joint was also measured throughout using a gyro

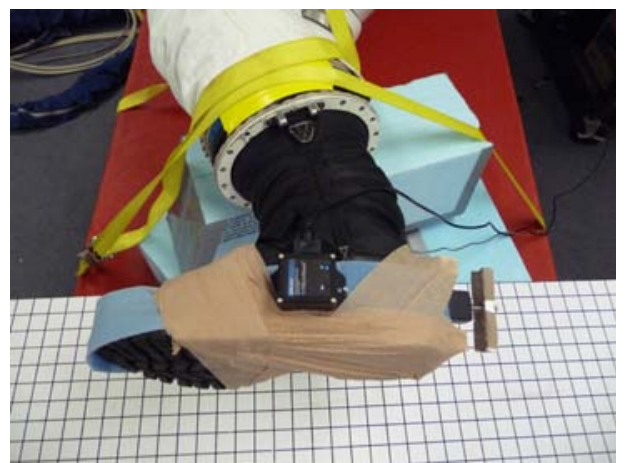

Figure 1. Joint torque test method setup. Load cell and orientation sensor attached to boot for ankle joint torque.

\footnotetext{
${ }^{1}$ Space Suit Engineer, Crew and Thermal Systems Division, 2101 NASA Parkway, Houston, TX 77058/EC5, AIAA Member.

${ }^{2}$ Extravehicular Activity Task Instructor, Mission Operations Extravehicular Activity Task Group, 2101 NASA Parkway, Houston, TX 77058/DX3.
} 
enhanced orientation sensor to capture where the torque values occurred in the joint's ROM. An example of the test setup can be seen in Fig. 1. Full details and results of the testing can be found in the "Space Suit Joint Torque Test Report." 1 Additional information with regards to the test method can be found in "A Method for and Issues Associated with the Determination of Space Suit Joint Requirements"” and also in "Results and Analysis from Space Suit Joint Torque Testing."3 The gyro enhanced orientation sensor is the "accelerometer" refered to in these reports.

The methodology developed to establish joint torque requirements was also mandated as the verification method for all joint torque requirements in the Constellation Program Extravehicular Activity (EVA) Systems Project Office (ESPO) Space Suit Element Requirements Document (ERD). ${ }^{4}$ However, there was some question as to whether this new joint torque method was repeatable enough to be considered a standard verification method. Thus, a representative subset of the previous test points was repeated in a second round of testing to validate the method itself. A new test conductor with no prior experience in joint torque testing completed the second round of testing; the new test conductor was only provided with the same information that would be available to space suit contractors during requirements verification testing. The previous test conductor was not physically involved in the setup or conduct of the second round of testing, but was available for consultation as would have been the case for Constellation space suit contractors. It was assumed that the method could then be considered valid if the resultant data from the second test series were comparable to the original test. If the data were not comparable, an attempt would be made to identify and eliminate the variables that could have caused the discrepancy between the two data sets. If necessary, additional testing would occur to evaluate the success of removing the variables and the overall repeatability of the method. Any additional test setup and execution details not included in the original description of the method would then be documented to ensure repeatability. This paper discusses a two-part effort made to validate the joint torque measurement method.

\section{Validation Test Data Collection}

A test conductor not familiar with the original test was selected to carry out part one of the two-part validation testing and is referred to throughout this paper as TC2. The information available to TC2, which would also be available to contractors developing future space suits, consisted of test plans, reports, photos, video, and discussions with the previous test conductor who will be referred to throughout this paper as TC1.

The original test consisted of determining joint torque values for the joints of several existing and prototype space suits. The validation test needed only to consist of a subset of these to demonstrate repeatability and therefore a specific suit and joint motions were selected. The Mark III prototype space suit was selected for the validation test because it has a combination of several different joint designs. Additionally, the Mark III was selected because all of its joints could be tested using the proposed joint torque measurement method, therefore allowing data comparisons between the two data sets to be made for the greatest number of joints. The Mark III was also readily available to be scheduled for testing. A challenge associated with use of the Mark III is that it has a lot of mobility built into its design, which makes it more difficult to isolate and position specific joints for testing. The subset of joint motions selected were: elbow flexion/extension, knee flexion/extension, shoulder adduction/abduction, shoulder flexion/extension, shoulder medial/lateral, hip flexion/extension, hip adduction/abduction, torso flexion/extension, and ankle dorsiflexion/plantar flexion. These joints and motions were selected due to their relative simplicity to complete a small set of data collection and data review.

The basic test setup used in the original test was also used for the validation test and is discussed in this paragraph. An example of the test setup can be seen in Fig. 2. The test was conducted both at a Constellationdesignated vent pressure ( $0.8 \mathrm{psid})$ and also at the nominal suit pressure (4.3 psid). The suit was tested without the Thermal Micrometeoroid Garment (TMG) or cover layer installed. The suit was arranged such that the joint being tested was manipulated in a plane parallel to the ground in an effort to eliminate the effects of gravity as much as possible. In most cases, the pressurized suit was laid on a lab table and supported as necessary by foam blocks to position the selected joint as desired. The suit was then securely restrained by ratchet straps to isolate the selected joint while keeping the rest of the suit stationary. The orientation sensor was attached on top of the moving portion of the selected joint to measure angular motion. The attachment was accomplished using hook and loop strips and/or residue-free tape to ensure no relative motion between the orientation sensor and suit. The load cell was attached to the end of the joint being tested such that it measured the force required to manipulate the joint through its ROM. Attachment of the load cell to the suit was similar to that used for the orientation sensor, and it was placed parallel to the ground and perpendicular to the axis of joint rotation. An initial joint angle measurement was taken prior to each data set trial to identify the starting position of the joint. The angular measurement was typically taken such that when the joint is aligned along the suit body axis, the angle measurement would be zero. A distance measurement was taken from the load cell to the axis of joint rotation to convert raw force data from the load cell into torque. 
Angle and distance measurements were based on the estimated position of a human joint within the space suit. Drag was eliminated from the tested joint as much as possible by either replacing items such as gloves with test plugs, and/or by supporting sagging areas using a tricaster rolling support. If desired, a grid board was placed under the joint being manipulated to provide a surface for the caster support to roll on, as a reference for measuring range of motion via overhead video, and for assistance in reviewing the data post-test. The grid board was supported by bar clamps and a tripod-style C-stand such as those used by the motion picture industry. The suit setup was documented via still photography, as well as overhead video. Each dataset was documented on the video by writing the joint, suit pressure, and trial number on a white board, which was pointed at the video camera for a few seconds

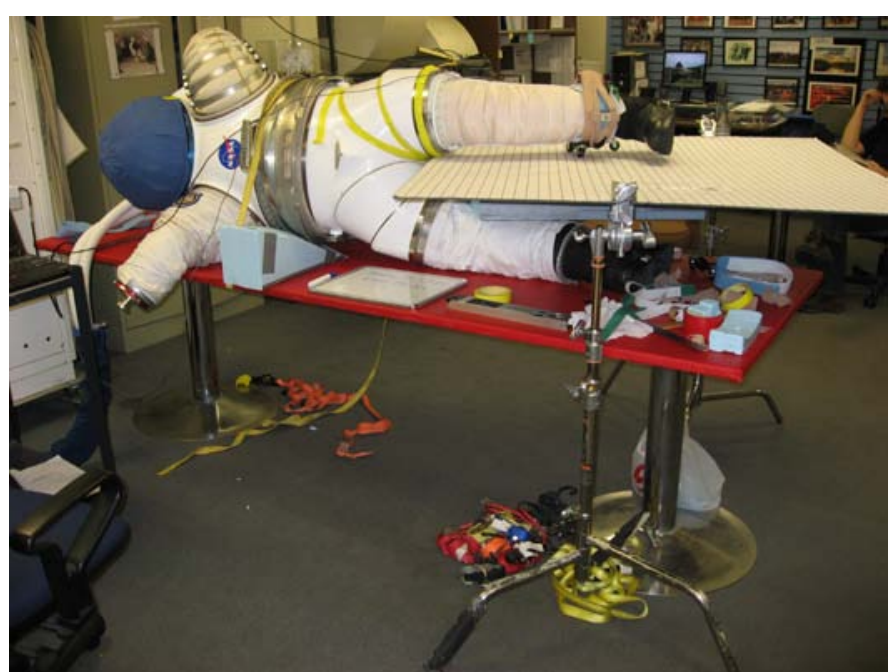

Figure 2. Test setup example. TC2 test setup for knee flexion/extension. prior to each test. Each joint was tested three times at both pressures, with 5 to 10 manipulations through the complete ROM in each of these three trials. Bearing joints were typically moved faster than non-bearing joints because the motion was easier, so more repetitions were necessary to collect enough datapoints.

The purpose of the validation test was to duplicate the test setup and joint motions of TC1 as best as possible using all of the information available from TC1. At the time of the first part of validation testing, video from the test for TC1 was not available for a portion of the subset of joint motions. When video was absent, TC2 used pictures and ROM reference figures as guides to attempt to complete the same motion with the same setup.

The entire subset of joint motions for the Mark III suit was able to be completed by TC2. During each trial, the starting angle as well as any notes associated with the test setup or test itself were documented.

Following the test by TC2, the raw data collected was processed and graphed in the same manner as the TC1 test method. Using the data processing techniques employed by TC1, TC2 used a spreadsheet to calculate joint torques from the raw data and to adjust the initial angle reported by the orientation sensor to match the measured pretest angle for each trial of each joint motion. The resultant values were plotted to show torque on the vertical axis and joint angle on the horizontal axis (see Fig. 3).

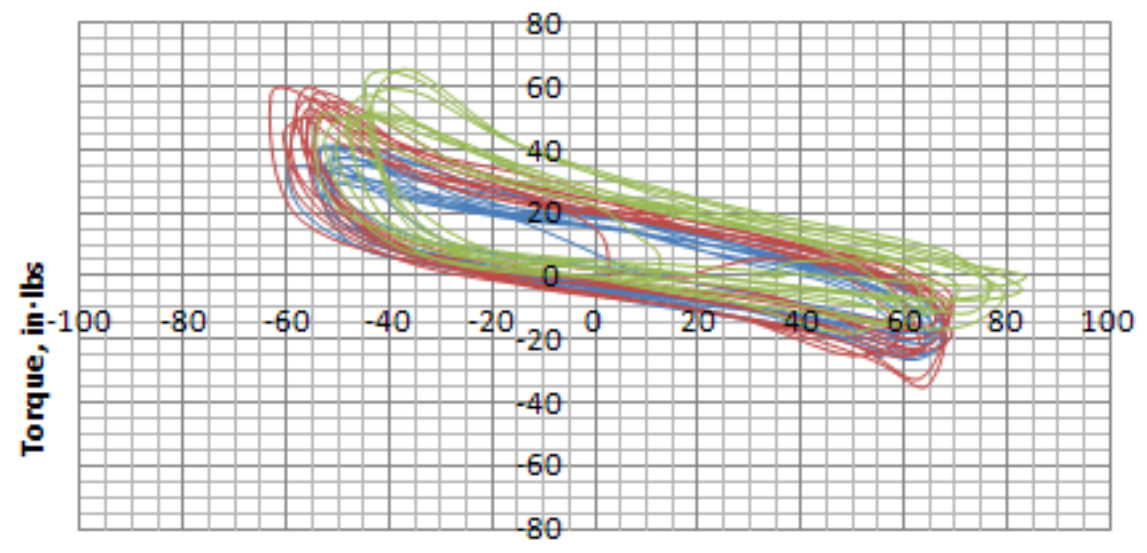

Angle, degrees

Figure 3. Resultant plot example. TC2 test for ankle dorsiflexion (positive angle)/plantar flexion (negative angle) at 4.3 psid. 


\section{Comparing Results}

\section{A. Analysis Methods}

Multiple methods of analysis were used to aid in the comparison process. The methods of analysis were generally categorized as either graphical or statistical analysis. These methods were chosen to provide visual comparisons as well as non-subjective statistical comparisons.

\section{Graphical Analysis}

The primary aim of the graphical analysis techniques was to provide rough visual comparisions between the torque-angle plots generated for each joint by each test conductor with focus on overall shape and maximums. To visually compare the plotted data, the traces for each joint motion and pressure to be compared from the two test conductors were adjusted to be on identical axes. They were then placed side by side, with each test conductor's graph consisting of the three trials designated by color, as seen in Fig. 4a-b (trial 1 - blue, trial 2 - red, trial 3 green).
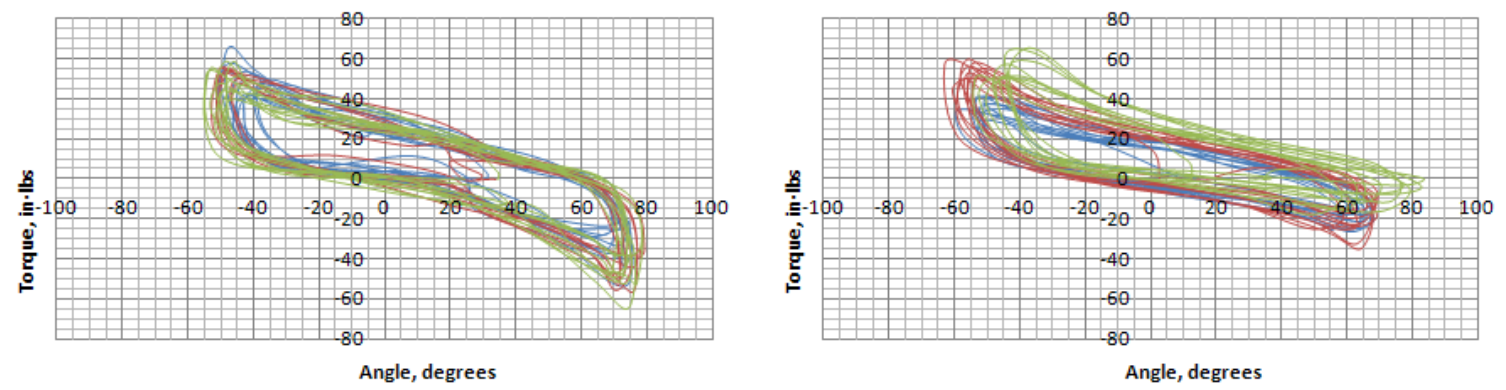

Figure 4a-b. Side-by-side graphical comparison. TC1 test (left) and TC2 test (right) for ankle dorsiflexion/plantar flexion at 4.3 psid.

A visual comparison was made by looking at deviations in patterns and torque values between the three trials within and across test conductors. In addition to the two side-by-side graphs, a third graph, overlaid with the traces of one trial from each test conductor, was used to allow direct trial-to-trial comparisons and to focus on the variance of torque values at the angle boundaries (see Fig. 5). Only one trial from each test conductor was selected to minimize the clutter of plotted lines. In most cases, Trial 3 was selected from each test conductor with the logic that the test conductor would be the most experienced with the joint motion for this trial. However, if an obvious discrepancy existed between Trial 3 and the first two trials, Trial 3 was considered an outlier trial and a more representative trial from the first two was selected. An additional feature of a ROM boundary was added to the third graph. This highlighted area of the graph represented the "reference ROM requirements" that were used in the "Space Suit Joint Torque Test Report." ${ }^{1}$ The purpose of the reference ROM requirements for that report were to aid in the selection of torque values, those which occurred at the ends of the angle boundaries, for Constellation requirements. The boundaries were used in the validation testing to replicate the potential process used to select torque values for future requirements.

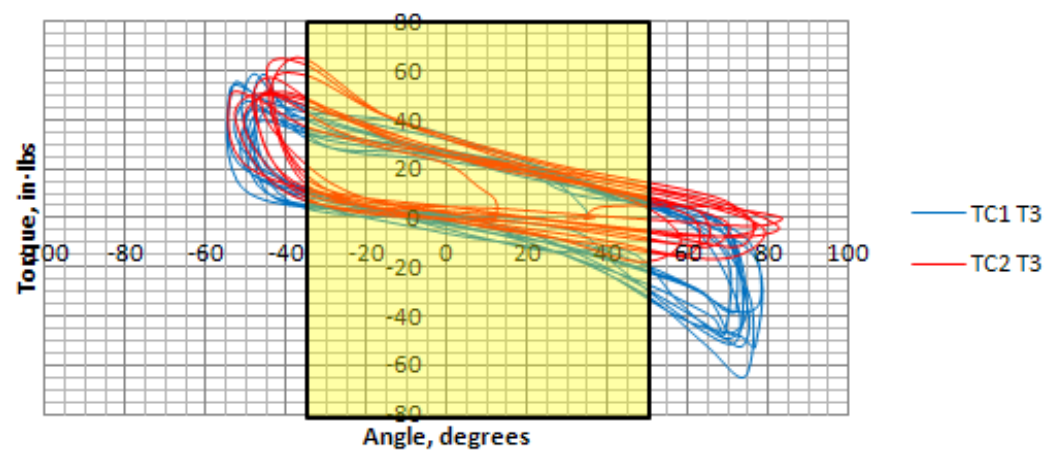

Figure 5. Overlapping graphical comparison. Trial 3 for both test conductors of ankle dorsiflexion/plantar flexion at 4.3 psid with designated ROM boundaries. 
In addition to the visual pattern comparison, a table was made in an attempt to highlight discrepancies between torque values (see Table 1).

Table 1. Joint Torque Comparison and Measurement Variables. Ankle dorsiflexion/plantar flexion at 4.3 psid.

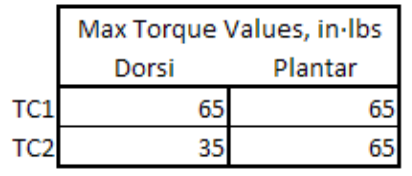

\begin{tabular}{|c|c|c|c|}
\hline \multicolumn{4}{|c|}{ Torque Values at ends of ROM, in.lbs } \\
\hline Dor (Min) & Dor (Max) & Pla (Min) & Pla (Max) \\
\hline 20 & 35 & 32 & 50 \\
\hline 10 & 25 & 20 & 35 \\
\hline
\end{tabular}

\begin{tabular}{|r|r|r|r|}
\hline \multicolumn{3}{|c|}{ Starting Angle (+ or - neutral) } & Distance, inches \\
\hline \multicolumn{1}{|c|}{$\mathrm{T} 1$} & \multicolumn{1}{c|}{$\mathrm{T} 3$} & \\
\hline 31 & 28 & 35 & 5.5 \\
\hline 8 & 3 & 10 & 5 \\
\hline
\end{tabular}

The table includes overall maximum torque, maximum and minimum torque at the designated ROM boundaries, measured starting angle for each trial, and measured distance from the location of the applied force to the axis of joint rotation. A visual estimation using the side-by-side graphs mentioned earlier was used to populate the torque values within the table. The overall maximum torque represented the maximum torque value plotted without taking into consideration the designated ROM boundaries. The maximum and minimum torque at the designated ROM boundaries were used to capture a band or range of torque values at the boundaries using all the trials for each test conductor. The values included in this band were only torque values produced for motion moving away from the neutral position, or zero angle, and did not include the return to neutral values that occurred when the motion direction was changed. This was done to capture the maximum torque values which generally occur during this type of motion, moving away from neutral. For example, the Dorsiflexion Torque Band, as seen in Fig. 6a, for the ankle included the torque values that occurred only when the joint motion was moving away from neutral position in the Dorsiflexion direction, as seen in Fig. 6b. The same was done for the Plantar Flexion Torque Band (Fig. 6a-b).

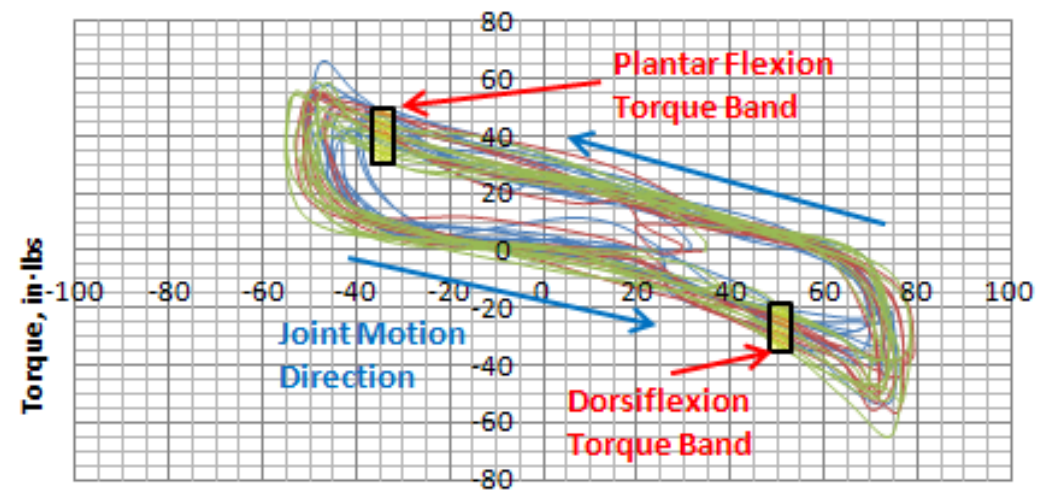

Angle, degrees

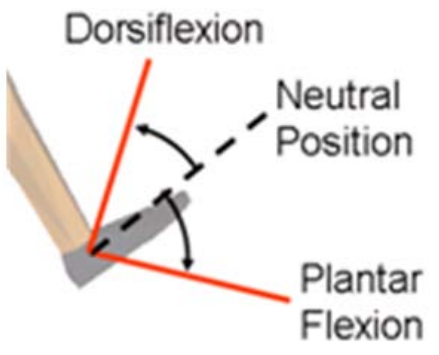

Figure 6a-b. Estimated torque bands. TC1 test for ankle dorsiflexion/plantar flexion at 4.3psid (left), and ankle ROM reference figure (right).

The measured starting angle was the pre-trial joint angle measurement taken to identify the starting position of the joint with respect to the neutral position of the ROM reference figures (see Fig. 6b). If the starting angle was considered on the plantar flexion side of neutral, for the ankle ROM reference figure, a negative angle value would be listed in the table. The starting angle and the measured distance were included in the table as references to identify if these variables had an effect on the plotted values.

\section{Statistical Analysis}

The second category of the analysis methods used was statistical analysis. This category of methods was used in an attempt to derive data that were not as subjective as the graphical analysis methods used. All of the data for the statistical analysis were extracted from the test conductors' data sets using spreadsheet formulas. The presence of a negative sign on the torque values represents whether the load cell is being pushed or pulled, therefore the sign on the torque values can be ignored for the statistical analysis.

Two approaches were taken to provide data to aid in the validation of the joint torque measurement method. The first approach compared maximum torque values between the two test conductors. The second approach focused on the repeatability of torque values within a single trial from a single test conductor. 
For the first approach, a table was made to capture the maximum torque value for each trial of each test conductor (see Table 2). This table includes the overall maximum torque and also the maximum torque that occurred within the designated ROM boundaries.

Table 2. Maximum Torque Value Comparison. TC1 and TC2 tests for ankle dorsiflexion/plantar flexion at 4.3 psid.

\begin{tabular}{|c|c|c|c|c|}
\hline \multicolumn{5}{|c|}{ Overall Maximum Torque, in $\cdot$ lbs } \\
\hline$\underline{T C 1}$ & Trial 1 & Trial 2 & Trial 3 & Average \\
\hline Dorsi & -52 & -55 & -65 & -57 \\
\hline Plantar[ & 66 & 55 & 59 & 60 \\
\hline$\underline{T C 2}$ & Trial 1 & Trial 2 & Trial 3 & Average \\
\hline Dorsi & -27 & -35 & -18 & -27 \\
\hline Plantar[ & 41 & 60 & 65 & 55 \\
\hline & & & & Variance \\
\hline & & & Dorsi[ & -30 \\
\hline & & & Plantar & 5 \\
\hline
\end{tabular}

\begin{tabular}{|c|c|c|c|c|c|}
\hline \multicolumn{6}{|c|}{ Maximum Torque within ROM, in $\cdot l b s$} \\
\hline$\underline{T C 1}$ & Trial 1 & Trial 2 & Trial 3 & Average & \multirow{3}{*}{$\begin{array}{r}\text { ROM Boundary } \\
35 \text { degrees }\end{array}$} \\
\hline \multirow{2}{*}{$\begin{array}{r}\text { Dorsi } \\
\text { Plantar }\end{array}$} & -25 & -21 & -30 & -25 & \\
\hline & 47 & 43 & 43 & 44 & \\
\hline \multirow{6}{*}{$\begin{array}{l}\frac{T C 2}{\text { Dorsi }} \\
\text { Plantar }\end{array}$} & Trial 1 & Trial 2 & Trial 3 & Average & \multirow{6}{*}{$\begin{array}{r}\text { ROM Boundary } \\
\begin{array}{r}50 \text { degrees } \\
35 \text { degrees }\end{array}\end{array}$} \\
\hline & -21 & -25 & -18 & -21 & \\
\hline & 31 & 39 & 58 & 43 & \\
\hline & \multicolumn{4}{|r|}{ Variance } & \\
\hline & & & Dors & -4 & \\
\hline & & & Plantar & 1 & \\
\hline
\end{tabular}

Within Table 2, the average of the trials for each test conductor was calculated and then compared between the test conductors to generate the delta variance.

The second approach took a look at how consistent the cycles were throughout a single trial. This was done by focusing on the bounded ROM torque values. The consistency of the bounded ROM torque values was analyzed by calculating the average and standard deviation (assuming a normal distribution) of the datapoints that occurred on or just before the designated ROM boundaries for the 5 to 10 cycles within a single trial (see Table 3 ). To offer a visualization, the datapoints used would be those that would fall in or just before the Torque Bands in Fig. 6a for a single trial. In addition to listing the average and standard deviation for the respective motion, Table 3 also lists the range in which the torque and angle values occured. This range is represented in Table 3 with the maximum and minimum titled values.

Table 3. Single Trial Torque and Angle Standard Deviation. Bounded ROM torque and angle maximum, minimum, average, and standard deviation for TC2 test, Trial 3 for ankle dorsiflexion/plantar flexion at 4.3 psid.

\begin{tabular}{|c|c|c|c|}
\hline \multicolumn{2}{|c|}{ Torque, in.lbs } & \multicolumn{2}{|c|}{ Angle, degrees } \\
\hline Dorsiflexion & Plantar Flexion & Dorsiflexion & Plantar Flexion \\
\hline Maximum & Maximum & Maximum & Maximum \\
\hline-17.8 & 57.9 & 49.6 & -34.7 \\
\hline Minimum & Minimum & Minimum & Minimum \\
\hline-0.5 & 28.5 & 22.9 & -5.3 \\
\hline Average & Average & Average & Average \\
\hline-7.6 & 38.1 & 40.4 & -19.2 \\
\hline Standard Deviation & Standard Deviation & Standard Deviation & Standard Deviation \\
\hline 5.0 & 8.8 & 8.1 & 9.6 \\
\hline
\end{tabular}

\section{B. Discussion}

All of the mobility features analyzed consisted of either softgoods, multiple bearings, or a combination of softgoods and bearings. The nature of these items in itself leads to inconsistancies in the required torque needed to manipulate the joint through its designed ROM. With softgoods, differences in how a single mobility feature folds or unfolds as it is cycled through its ROM could lead to different amounts of torque required to move it through that same ROM each time. The pattern of the softgood is designed to minimize this randomness in most cases. For multiple bearings, differences in how each bearing rotates in relation to the other bearings as the assembly is cycled through its ROM could also lead to a difference in torque values. With this being known, an attempt to identify other potentially controllable sources of deviations was made. 
The analysis performed to compare the two sets of test data revealed that variances did occur. The variances that occurred were generally in torque and angle values, yet in most cases the graphical pattern was able to be repeated. Some types of motions produced greater variances than others. The types of motions that produced the greatest variances were complex motions that involved multiple mobility features or occurred on multiple axes or a combination of both of these factors. These types of motions tended to produce variances in values and, in some cases, pattern (see Fig. 7).

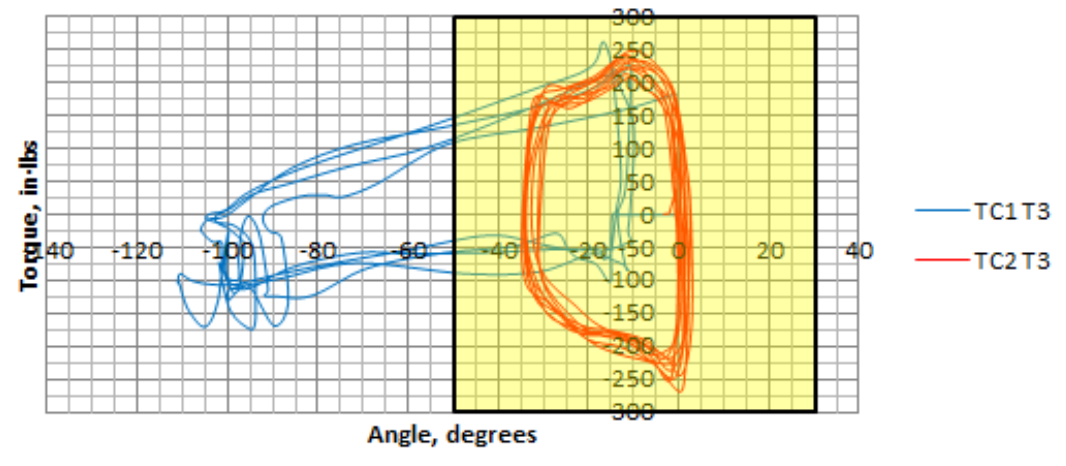

Figure 7. Complex motion variances. Trial 3 for both test conductors of hip adduction/abduction at 4.3 psid with designated ROM boundaries.

The simple joint motions - those that involved a single mobility feature and on a single axis - typically produced similar graphical patterns between the two data sets and may or may not have varied in torque and angle values (see Fig. 8).

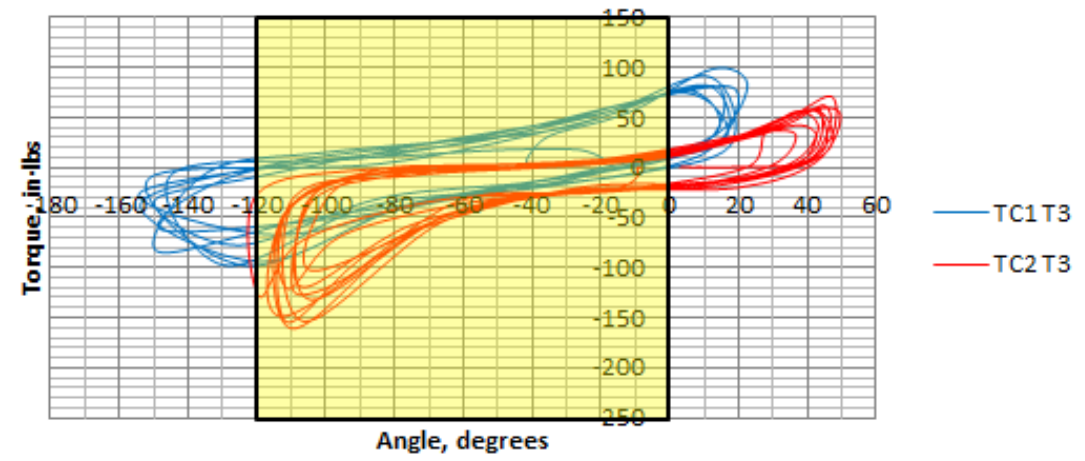

Figure 8. Simple motion variances. Trial 3 for both test conductors of knee flexion/extension at 4.3 psid with designated ROM boundaries.

Potential sources of deviations were identified to explain the variances in values and patterns. First, a human is pushing and pulling the load cell. This can result in inconsistent cycling of the joint and potential error in measuring the total force used, as seen by the load cell. The highest torques occur at the extremes of the ROM of the joint. Therefore, the overall maximum torque values of a trial can be influenced by the extent to which the extremes are reached during the cycling. Also, applying an indirect force into the load cell with the handle, resulting in side loading of the handle, may generate error in measuring the total force used. Second, a human is attempting to measure the starting angle of the joint in relation to the ROM reference figures. Variance of this reading translates the graph left or right in relation to the zero angle position. This would have a direct effect on the torque values that would fall within the reference ROM requirement boundaries, if used. Third, deviations in test setup and the path of the joint motion could have led to variances in values and patterns. In a few instances, video was not available to TC2 to observe the path of the joint motion that TC1 produced, which resulted in different interpretations of the joint motion between the two test conductors. Fourth, the rate at which the joint was cycled had a direct impact on the amount of gap in angle values between datapoints, as data were recorded at a constant rate, which in turn affects which torque values are recorded. Lastly, "accelerometer drift" (see Fig. 9 green trace line), as refered to by TC1, occurred in some instances within both sets of data, not necessarily for the same joint motion, and was thought to be caused by insecure attachment of the orientation sensor to the joint being rotated. This affected the angle reading and translated the graph left or right in relation to the zero angle position. 


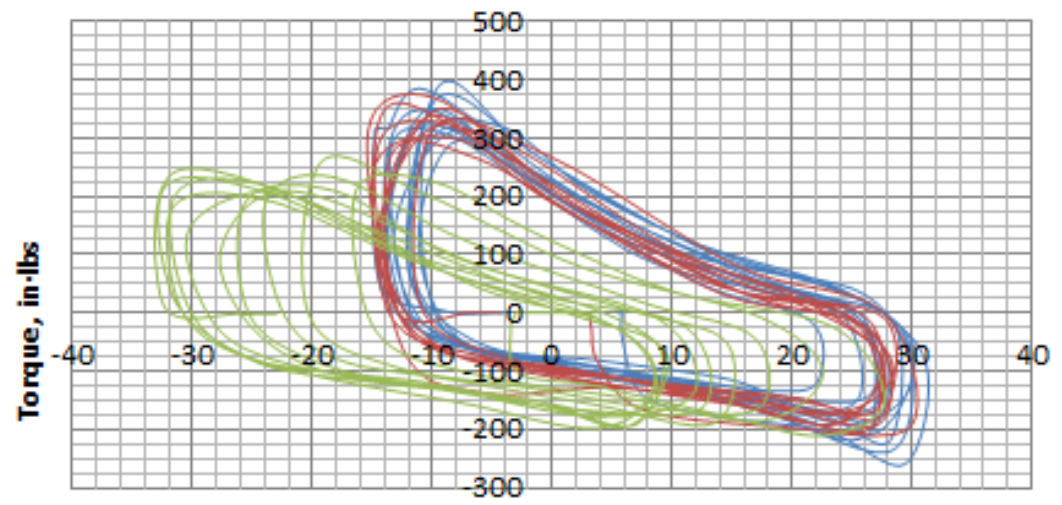

Angle, degrees

Figure 9. Accelerometer “drift.” TC2 test for torso flexion/extension at 4.3 psid.

\section{Validation Testing - Part Two}

Following the identification of the potential sources of deviations from part one of the validation testing, it was determined that the method could not be validated at that point and additional testing by another test conductor, TC3, was performed in an attempt to eliminate or minimize the effects of the variables. The same suit and subset of joint motions used in part one of the validation testing was used for part two with the exception of shoulder flexion/extension and shoulder medial/lateral. These two motions were excluded because in either case, the mobility feature on the Mark III consisted solely of a rotational bearing which was torque tested pre-delivery; the torque values were assumed to be unchanged between the configurations. At the conclusion of the second part of the validation testing, the data was compared to the previous two sets of data. Again, a determination was made as to whether the method could be validated or whether additional research was needed. Any changes to the test setup and execution of the joint torque measurement method found to improve the repeatability of the results were documented.

\section{A. Modifications and Additions}

For the second part of the validation testing a focus on answering the following questions, in addition to the repeatability question, was made to better understand the affects of the method:

1. Will more frequent data points better represent the torque values and angles?

2. Can load cell movement and "accelerometer drift" be minimized?

3. Can the starting angle be obtained in an easier, more accurate manner?

4. Does the angle that a test conductor pulls or pushes on the load cell handle (side load) affect torque value?

5. How sensitive is the force value to motion speed?

6. How does static positioning affect torque?

7. Does deviation occur when a different test conductor (TC4) uses the exact same setup as another test conductor (TC3)?

Several modifications and additions were then made to the original method for part two of the validation testing to aid in answering the questions.. One modification made was to attempt more stable load cell and orientation sensor attachment in order to provide more accurate readings and eliminate "accelerometer drift". A second modification was to start the joint at the zero angle position, as interpereted by the test conductor using the ROM reference figures, to eliminate starting angle measurement error. Seven additional trials were added to the three regular trials used in the method. A description of these trials and the motivation behind performing them is listed in Table 4 .

Table 4. Part Two - Additional Trials

\begin{tabular}{|l|l|}
\multicolumn{1}{|c|}{ TRIAL DESCRIPTION } & \multicolumn{1}{c|}{ MOTIVATION } \\
\hline Intentionally side-load the load cell handle & Understand load cell accuracy with human operation \\
\hline Perform cycles of trial at twice the rate of the regular trials & Understand effects of cycle rate on data collection and load cell accuracy \\
\hline Perform cycles of trial at half the rate of the regular trials & Understand effects of cycle rate on data collection and load cell accuracy \\
\hline Pause motion at 10 degree increments throughout cycling & Understand effects of static force on data collection and load cell accuracy \\
\hline Perform 3 regular trials with fourth test conductor (TC4) using TC3's test setup & Understand effects of test setup \\
\hline
\end{tabular}




\section{B. Data Collection}

The entire subset of joint motions for part two of the validation testing was able to be completed. Both TC3 and TC4 were able to conduct three regular trials for each of the joint motions. During each trial, any notes associated with the test setup or test itself were documented. The stabilization of the load cell and orientation sensor occurred by adding additional tape to the unit itself and to its data cable. However, during testing, it was noted that the orientation sensor angle data would periodically need additional stabilization time. The angle data appeared to be drifting at the start of some of the trials while being held in the static zero angle position and during the first few cycles of joint motion. Therefore, the count of 5 to 10 cycles needed to complete a trial was not initiated until the angle data appeared to no longer be drifting. In regards to the second modification described in part IV.A., the joints were able to be held by the test conductor in the zero degree position as interperated by the test conductor at the start of the data collection. As for the additional trials added, the side-loading of the load cell handle and the double and half rate cycling were able to be conducted as interpereted by TC3. A grid board with 10-degree increments labeled on the board was used to perform the pause motion cycling by TC3. The increment cycling proved difficult to interpret and replicate by TC3 for some joint motions by using the board and therefore was not used for every subset motion listed in part two of the validation testing. Following the test, the raw data collected was processed and graphed in the same manner as the TC1 test method.

\section{Discussion}

To compare the results of the trials conducted by TC3 and TC4 to those by TC1 and TC2, the graphical analysis method described in section II.A.1. was used. After using this method to compare the results and to conduct further analysis of the affects of the modified fish-scale method, the graphical analysis method was deemed to be sufficient enough to answer many of the questions identified in part two of the validation testing. The statistical analysis method used in section II.A.2. provided additional justification that further testing was needed from part one of the validation testing, however it was deemed to not be as applicable for part two of the validation testing. Instead, a more in-depth analysis using statistical analysis software will be used in forward work to attempt to identify the extent of the effect that each known source of deviation creates in the modified fish-scale method.

The first graphs produced were the test conductor single trial overlapping comparison style graphs (see Fig. 10)

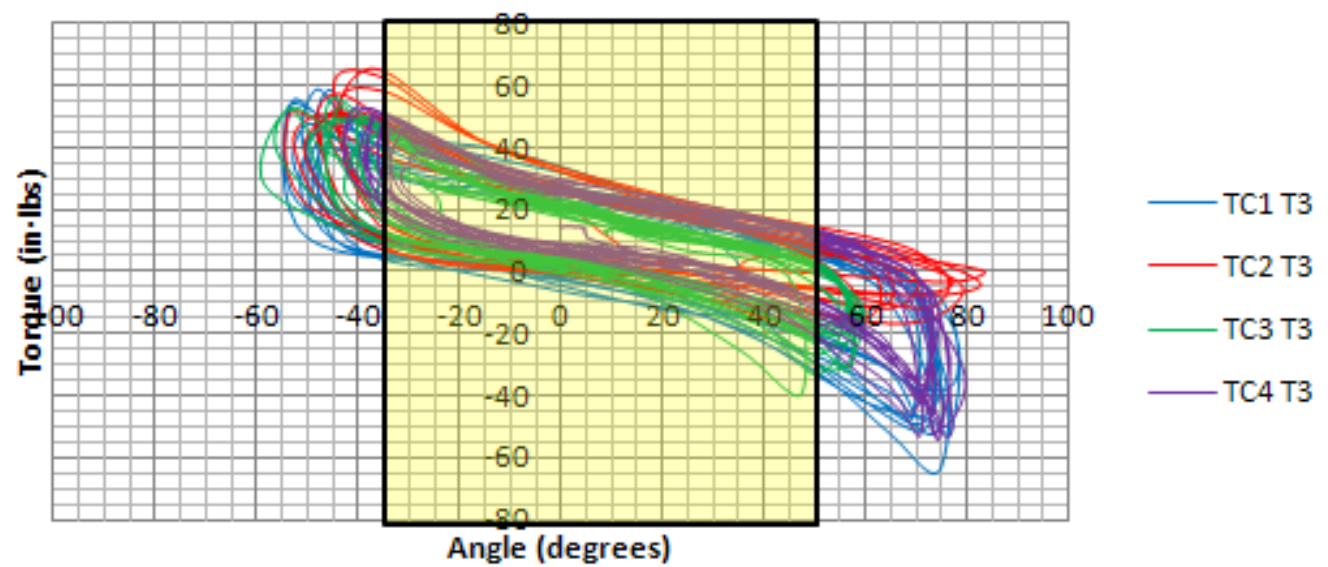

Figure 10. Overlapping graphical comparison. Trial 3 for all test conductors of ankle dorsiflexion/plantar flexion at 4.3 psid with designated ROM boundaries.

These graphs further confirmed that the pattern of the joint motion could be repeated and that similar torque values could be accomplished through the modified fish-scale method. Even though it was evident from the graphs that deviations in torque values, even within a single trial, did still exist to some extent, for the intention of the use of this joint torque measurement method, these deviations will be considered acceptable and any methods used to minimize these deviations that are not already included in the procedures will be added.

The graphs also confirmed that further work is still needed to produce repeatable angle measurements and the zero angle position (see Fig. 11). 


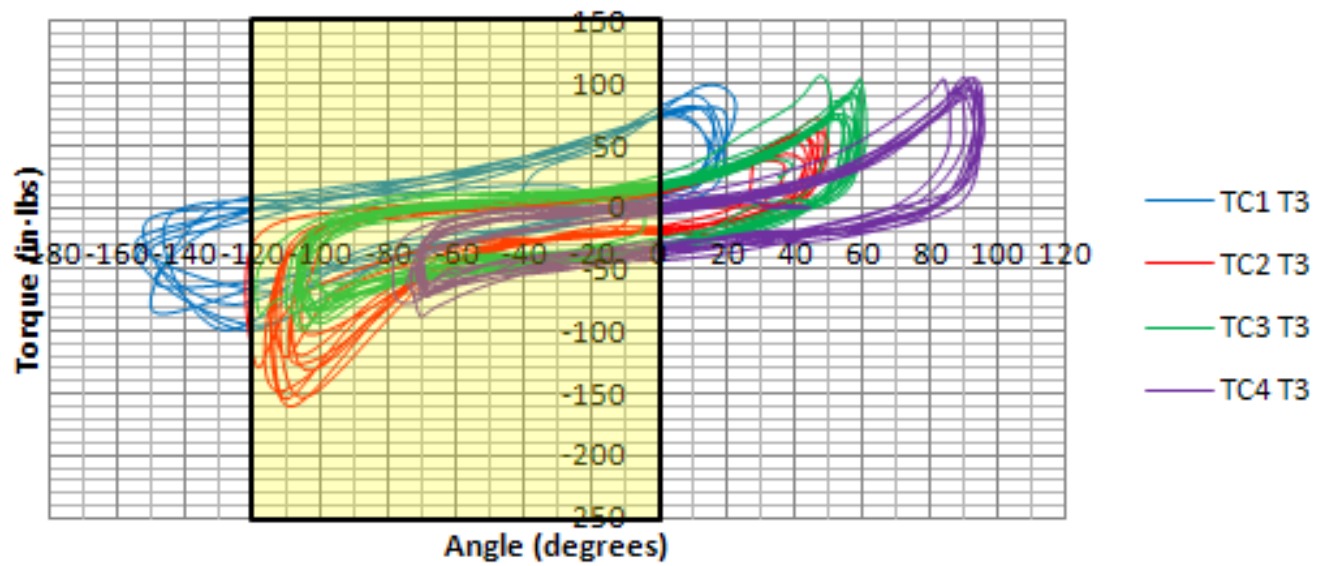

Figure 11. Overlapping graphical comparison showing zero angle position deviations. Trial 3 for all test conductors of knee flexion/extension at 4.3 psid with designated ROM boundaries.

Despite an attempt made during the testing process to allow the angle drift to stabilize, angle drift and a deviation in starting zero angle position and ending zero angle position still existed in several trials. The source of this deviation, previously thought to be a loosely attached orientation sensor, was then concluded to be a function of the orientation sensor itself and how it generates angle data. A separate test was conducted on the orientation sensor to reproduce angle drift. The sensor was rotated through a similar ROM throughout the test, however the rate of rotation was greatly increased in the second half of the test. The plot of the results can be seen in Fig. 12 and shows that angle drift was created from the increased rate of rotation.

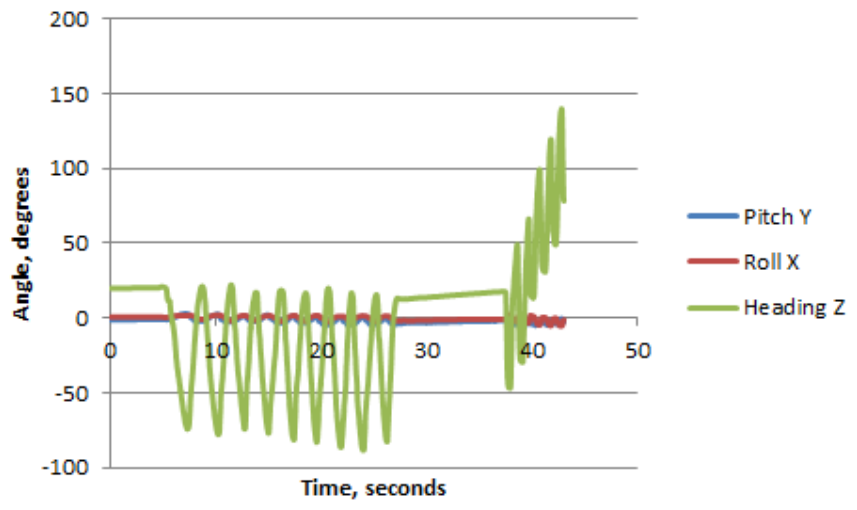

Figure 12. Orientation sensor test. The heading $Z$ plot represents the angle value used in the method .

The starting zero angle position can be adjusted during the data processing stage, however a method to prevent the angle drift during joint motion has not yet been identified for this joint torque measurement method. Forward work will be to identify an improved angle measurement method. One potential method could be the use of a motion capture system. Until that occurs, the current method using an orientation sensor is considered acceptable and any methods used to minimize these deviations that are not already included in the procedures will be added.

Answers to all of the additional questions identified in section IV.A. are addressed in the following paragraphs. The first question attempts to address the gap in data points which affects the torque and angle values recorded. An initial review of the program written to collect data from data acquisition system showed that the data recording speed was at a maximum for the types of equipment being used. For part two of the validation testing, no attempt to increase the data recording speed was made.

The second and third questions are partially related to the discussion held earlier in this section about load cell and orientation sensor attachment and angle measurements. Load cell and orientation sensor attachment can be improved through the use of additional residue-free tape. The starting angle can be obtained in an easier manner by 
holding the joint in the zero angle position at the start of each trial. However, the "accelerometer drift" was not eliminated and the zero angle position can still be interpereted differently between test conductors.

The fourth question addresses the potential of side-loading the load cell. After plotting the data obtained from the trials conducted by TC3 that intentionally input dramatic side-loading into the load cell handle and comparing them to one of the first three trials, it was evident that when side-loading occurs a portion of the total force required to manipulate a joint through its ROM is not realized and reported by the load cell. Therefore, the plot of the side-load trial could show lower torque values than the plots of the trials that attempt to apply direct force into the load cell (see Fig. 13).

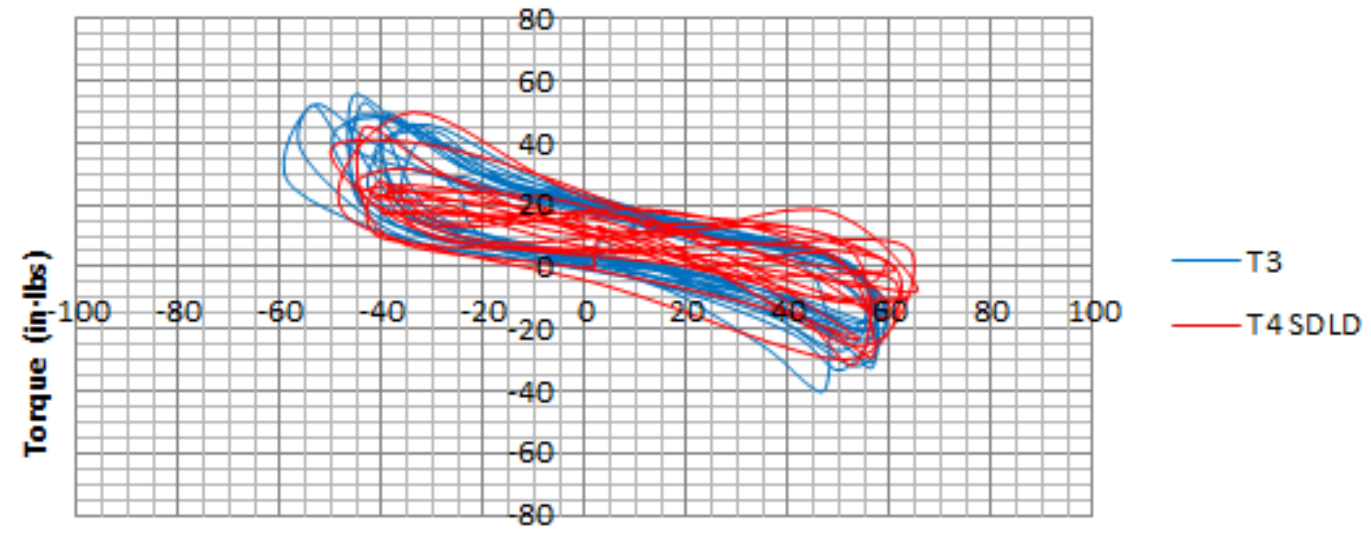

\section{Angle (degrees)}

Figure 13. Affects of side-loading. Trial 3 (normal motion) and Trial 4 (side-loading) for TC3 of ankle dorsiflexion/plantar flexion at 4.3 psid.

The fifth question looks at if the force value is sensitive to motion speed. The data from the double speed and half speed trials were plotted along with a normal speed trial to allow a comparison (see Fig. 14).

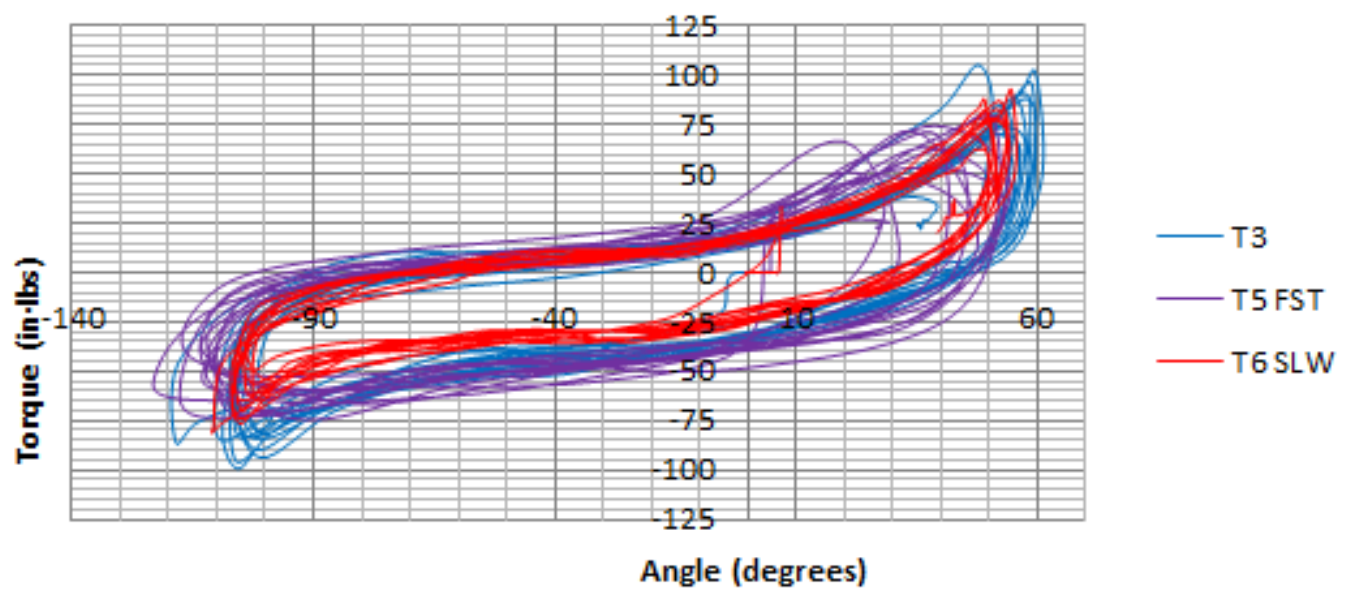

Figure 14. Affects of motion speed. Trial 3 (normal speed), Trial 5 (double speed), and Trial 6 (half speed) for TC3 of knee flexion/extension at 4.3 psid.

The general conclusion was drawn that the slow (half speed) trial produced torque values that were equal to or less than the torque values from the normal trial. It was also concluded that the fast (double speed) trial produced torque values that were equal to or greater than the normal trial. There can be a variety of explanations for this, one theory being that the speed of the joint causes an equal volume of air to displace at different rates which directly affects the torque values. Whatever the cause, it was apparent that the speed of the motion can have an effect on the torque values reported. Another observation from the visual comparison of the plots was that as the speed of the motion was increased, the uniformity of the torque values decreased. It will be noted in the method to perform the joint motion at a rate similar to that of the previous test conductors. 
The sixth question looks at how pausing the motion at a specified angle increment to generate a static force affects the torque values. The graphical analysis provided a variety of results for this comparison between the various joint motions and a clear conclusion could not be made. In general, the overall maximum torque values recorded for the static measurement trials were similar to those of the normal trials, however the angles at which those occurred did not necessarily match (see Fig. 15). Also, the torque values that were in between the overall maximums were less than those in the normal trials. In some cases where a caster was used to support the joint during the motion, the friction created by the caster was more than the required force to hold the joint at the incremented angle. When this was not the case, a couple of theories were presented as to why the torque values were less. The first being that static measurements do not take into account the force required to displace the air within the suit. The second being that the design of the joint could have incorporated a stay in place mobility feature. With this feature, the joint tends to stay in the position that it is moved to instead of tending back to it's neutral position.

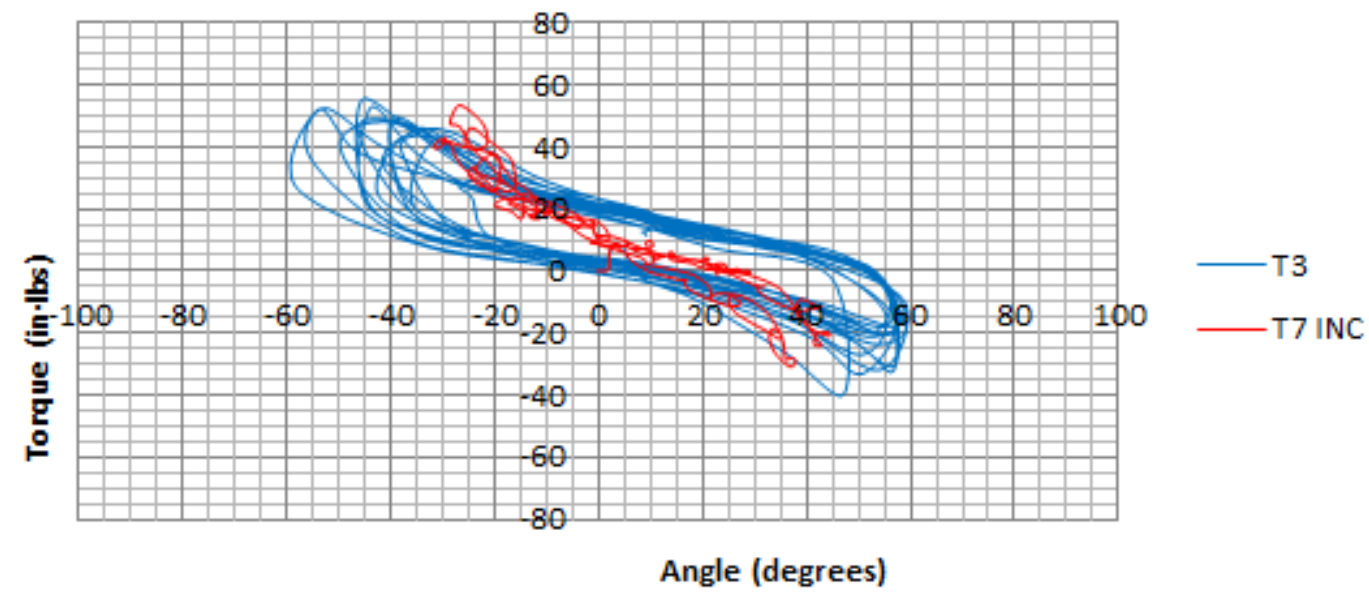

Figure 15. Affects of static measurements. Trial 3 (normal motion) and Trial 7 (incremental motion) for TC3 of ankle dorsiflexion/plantar flexion at 4.3 psid.

The final question looked at potential deviations in torque and angles directly contributable to different test conductors performing the test. The deviations that could occur from not having the exact same setup to perform the joint motions were eliminated in this comparison. The graphical comparison confirmed that the pattern could be repeated and similar torque values could be accomplished, yet deviations still occurred (see Fig. 16). It was concluded that many variables, as discussed in the preceding paragraphs, can affect the torque and angle values and that an exact duplication of these values using this method is impossible to achieve. As mentioned at the beginning of the discussion for part two of the validation testing, the deviations are considered acceptable.

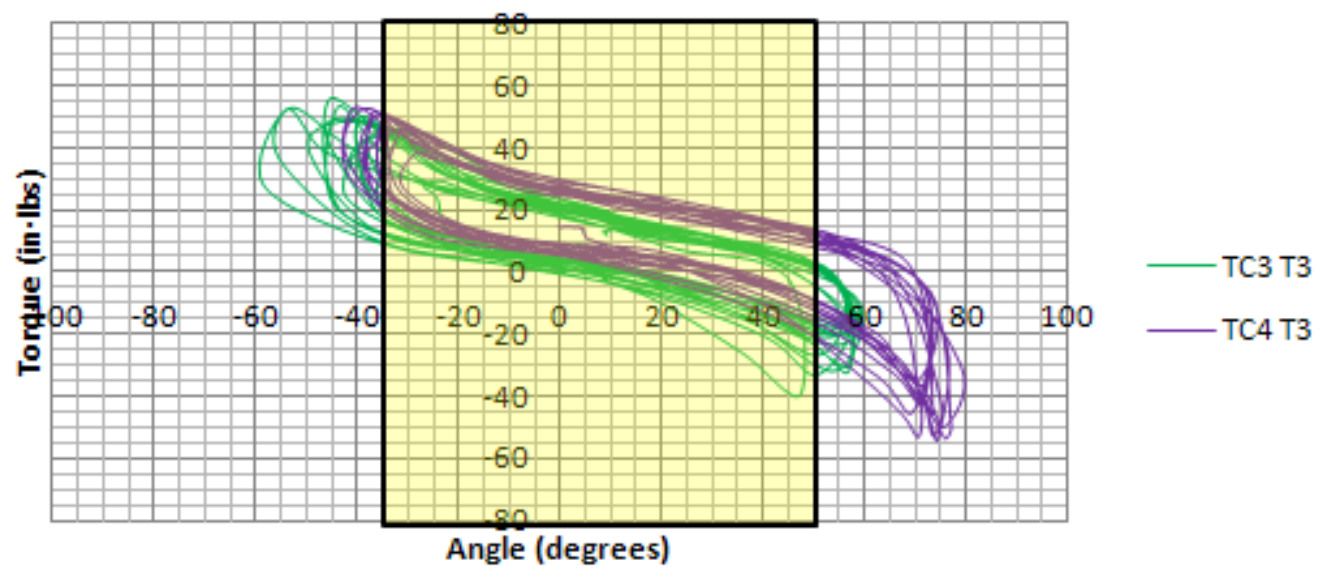

Figure 16. Affects of different test conductors. Trial 3 for TC3 and TC4 of ankle dorsiflexion/plantar flexion at 4.3 psid with designated ROM boundaries. 


\section{Changes to the Method}

A few minor changes to the method will be implemented for future joint torque testing using the modified fishscale method. The joint will be held by the test conductor for approximately 15 seconds in the zero degree position, according to the ROM reference figures, at the start of the data collection. This will be done without the test conductor holding the load cell handle. This provides the ability to zero-out the torque and angle values during the data processing stage. Additionally, the test conductor will provide a method to physically mark the zero degree position used at the beginning of the first trial so that following trials can all be started at the same zero degree position. Lastly, a note will be added to the method to highlight the importance of applying direct force to the load cell to minimize force deviations caused by side-loading the load cell.

\section{E. Forward Work}

As mentioned in the preceding discussion, the modified fish-scale method is an acceptable method to record joint torque values for space suit joints. However, as with many methods, there remain areas for improvement. Going forward, an in-depth statistical analysis will be conducted of the data used in the validation process in an attempt to gain a better understanding of the affects of the identified variables. An initial look at the potential of this analysis was conducted and can be seen in Fig. 17.

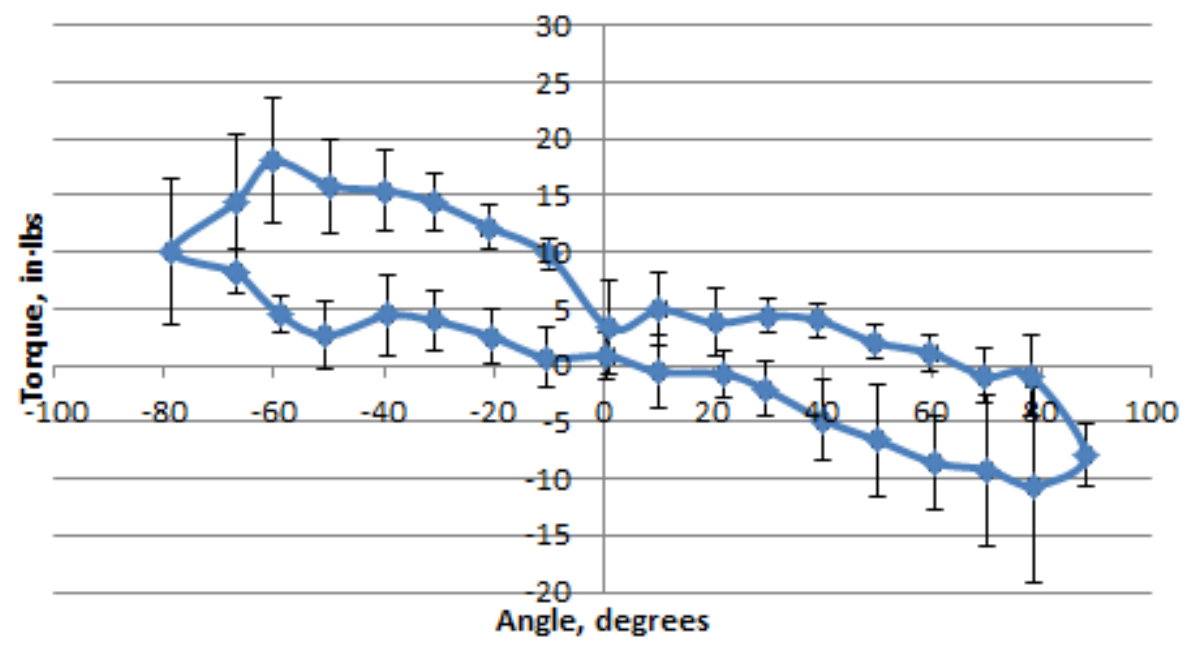

Figure 17. In-depth statistical analysis example. Combined data for all trials of all test conductors of ankle dorsiflexion/plantar flexion at 4.3 psid.

This graph was produced by combining the data of all of the trials by all of the test conductors for a single joint motion. Using this data, the average torque value that occurred over an angle range and the standard deviation over that range was calculated using a simple spreadsheet formula with the assumption that the data is normally distributed. The average torque value was plotted and the standard deviation of the population was represented using error bars. This example shows that the torque values at the ROM extremes have a potentially higher standard deviation than the rest of the torque values. A potential explaination for this is that changes in the angle at the ROM extremes produce increasingly higher torque values, therefore a torque value from an angle at the beginning of the angle range at the ROM extremes will be much less than a torque value from an angle at the end of the same angle range. On the other hand, by going back through the data, it was seen that in some cases the torque values began to decrease just prior to the motion changing direction. One possible explanation for this is that the joint possesses momentum and continues in one direction just prior to being forced to move in the opposite direction. As mentioned, this is just an example of how the statistical analysis could lead to a better understanding of the data produced by this joint torque measurement method. The results of the in-depth statistical analysis will be combined with the complete set of results from all of the analysis methods mentioned throughout this paper to produce a NASA Johnson Space Center, Crew and Thermal Systems Division document.

Additional work that could be conducted to improve the modified fish-scale method would be to improve the angle measurement method, potentially by using a motion capture system, and to increase the data recording rate. 


\section{Conclusion}

Accurately measuring the torque required to manipulate a joint on a pressurized space suit has proven to be a difficult task. The modified fish-scale method as described in the "Space Suit Joint Torque Test Report"1 was developed in an attempt to accomplish this task. However, a question of repeatability of the method arose and in response a validation process began. Initial testing as part of the validation process revealed that variances could occur and this led to the identification of potential sources of the deviations. A second set of validation testing occurred in an attempt to eliminate or minimize the effects of the variables. Observations made during this second set of validation testing showed that the use of a gyro enhanced orientation sensor could lead to inaccurate angle readings. Other sources of deviations were observed and noted. The conclusion was made that for the intended use of this joint torque measurement method, the method is accepted and validated as a repeatable process.

\section{References}

${ }^{1}$ Matty, J., “Space Suit Joint Torque Test Report,” NASA CTSD-CX-5304, 2010.

${ }^{2}$ Matty, J., and Aitchison, L., "A Method for and Issues Associated with the Determination of Space Suit Joint Requirements,” 2009-01-2537 SAE International, 2009.

${ }^{3}$ Matty, J., "Results and Analysis from Space Suit Joint Torque Testing,” AIAA 2010-6211, 2010.

${ }^{4}$ NASA, Constellation Program Extravehicular Activity (EVA) Systems Project Office (ESPO) Space Suit Element Requirements Document (ERD), CxP 72208, 2009. 